\title{
UCRL-PROC-223278
}

LAW RENCE LIVERMORE N A T IO N A L LABORATORY

\section{Bulk Properties of Iron Isotopes}

E. Algin, A. Schiller, A. Voinov, U. Agvannluvsan, T. Belgya, L.A. Bernstein, C.R. Brune, R. Chankova, P.E. Garrett, S.M. Grimes, M. Guttormsen, M. Hjorth-Jensen, M.J. Hornish, C.W. Johnson, T. Massey, G.E. Mitchell, J. Rekstad, S. Siem, W. Younes

July 31, 2006

International Conference on Nuclear Structure and Related Topics

Dubna, Russia

June 13, 2006 through June 17, 2006 
This document was prepared as an account of work sponsored by an agency of the United States Government. Neither the United States Government nor the University of California nor any of their employees, makes any warranty, express or implied, or assumes any legal liability or responsibility for the accuracy, completeness, or usefulness of any information, apparatus, product, or process disclosed, or represents that its use would not infringe privately owned rights. Reference herein to any specific commercial product, process, or service by trade name, trademark, manufacturer, or otherwise, does not necessarily constitute or imply its endorsement, recommendation, or favoring by the United States Government or the University of California. The views and opinions of authors expressed herein do not necessarily state or reflect those of the United States Government or the University of California, and shall not be used for advertising or product endorsement purposes. 


\section{Bulk Properties of Iron Isotopes}

E. Algin,${ }^{1,}{ }^{*}$ A. Schiller, ${ }^{2}$ A. Voinov, ${ }^{3}$ U. Agvaanluvsan,${ }^{4}$ T. Belgya,${ }^{5}$ L.A. Bernstein,${ }^{6}$ C.R. Brune,${ }^{3}$ R. Chankova,${ }^{7}$ P.E. Garrett,${ }^{6}$ S.M. Grimes, ${ }^{3}$ M. Guttormsen, ${ }^{7}$ M. Hjorth-Jensen, ${ }^{7}$ M.J. Hornish, ${ }^{3}$ C.W. Johnson, ${ }^{8}$ T. Massey, ${ }^{3}$ G.E. Mitchell,${ }^{9}$ J. Rekstad,${ }^{7}$ S. Siem,${ }^{7}$ and W. Younes ${ }^{6}$

${ }^{1}$ Department of Physics, Eskisehir Osmangazi University, Meselik 26480, Turkey ${ }^{2}$ NSCL, Michigan State University, East Lansing, MI-48824

${ }^{3}$ Department of Physics and Astronomy, Ohio University, Athens, OH-45701

${ }^{4}$ North Carolina State University, Raleigh, NC-27695

Triangle Universities Nuclear Laboratory, Durham, NC-27708

Lawrence Livermore National Laboratory, L-414, Livermore, CA-94551

${ }^{5}$ Institute of Isotope and Surface Chemistry, HAS, H-1525 Budapest, Hungary

${ }^{6}$ Lawrence Livermore National Laboratory, L-414, Livermore, CA-94551

${ }^{7}$ Department of Physics, University of Oslo, N-0316 Oslo, Norway

${ }^{8}$ San Diego State University, San Diego, CA-92182

${ }^{9}$ North Carolina State University, Raleigh, NC-27695

Triangle Universities Nuclear Laboratory, Durham, NC-27708

Nuclear level densities and radiative strength functions (RSF) in ${ }^{56} \mathrm{Fe}$ and ${ }^{57} \mathrm{Fe}$ were measured using the ${ }^{57} \mathrm{Fe}\left({ }^{3} \mathrm{He}, \alpha \gamma\right)$ and ${ }^{57} \mathrm{Fe}\left({ }^{3} \mathrm{He},{ }^{3} \mathrm{He}^{\prime} \gamma\right)$ reactions, respectively, at Oslo Cyclotron Laboratory. A low-energy enhancement in the RSF below $4 \mathrm{MeV}$ energy was observed. This finding cannot be explained by common theoretical models. In a second experiment, two-step cascade intensities with soft primary transitions from the ${ }^{56} \mathrm{Fe}(\mathrm{n}, 2 \gamma)$ reaction were measured. The agreement between the two experiments confirms the low-energy enhancement in the RSF. In a third experiment, the neutron evaporation spectrum from the ${ }^{55} \mathrm{Mn}(\mathrm{d}, \mathrm{n})^{56} \mathrm{Fe}$ reaction was measured at 7$\mathrm{MeV}$ deuteron energy at John Edwards Accelerator Laboratory at Ohio University. Comparison of the level density of ${ }^{56} \mathrm{Fe}$ obtained from the first and third experiments gives an overall good agreement. Furthermore, observed enhancement for soft $\gamma$ rays is supported by the last experiment. 


\section{INTRODUCTION}

Determination of nuclear level densities (NLDs) and radiation strength functions (RSFs) has been of great importance, in particular, in reaction modeling calculations. The experimental data on NLDs for many nuclei are obtained from the analysis of low-lying discrete levels and neutron resonance data. This information on NLDs covers a rather narrow excitation energy range. Therefore, other methods are necessary to determine the NLD in a wider ranges of excitation energies. One method, the so called Oslo method, has been proven to be valuable in determining both the NLDs and RSFs simultaneously from primary $\gamma$ spectra of a light-ion reaction. This method is based on the Axel-Brink hypothesis which assumes that the RSF depends only on the $\gamma$ energy and not on the excitation energies of the initial and final states. This assumption has been proven to be violated at sufficiently high temperatures $(\geq 1-2 \mathrm{MeV})$. Especially, the width of the giant dipole resonance has been shown to depend on temperature. Therefore, it is important to investigate the validity of the Axel-Brink hypothesis employed in the Oslo method.

Recently, the NLDs and RSFs for ${ }^{56} \mathrm{Fe}$ and ${ }^{57} \mathrm{Fe}$ nuclei have been extracted from ${ }^{57} \mathrm{Fe}\left({ }^{3} \mathrm{He}, \alpha \gamma\right)$ and ${ }^{57} \mathrm{Fe}\left({ }^{3} \mathrm{He},{ }^{3} \mathrm{He}^{\prime} \gamma\right)$ reactions using the Oslo method. An anomalous enhancement for soft transitions in the RSFs of these isotopes has been found. Furthermore, there is no present theory which can explain this enhancement. In order to ensure this enhancement we performed a second experiment using the ${ }^{57} \mathrm{Fe}(n, 2 \gamma)$ reaction. The two step cascade intensities extracted from this experiment confirmed the enhancement observed in the RSF of the ${ }^{57} \mathrm{Fe}$ nucleus obtained from the Oslo experiment.

Another method to extract the NLD below the particle separation energy has been the study of particle evaporation spectra from nuclear reactions. Due to the concerns related to the applicablity of the Axel-Brink hypothesis, we performed ${ }^{55} \mathrm{Mn}(d, n){ }^{56} \mathrm{Fe}$ reaction. The neutron evaporation spectra were analyzed, and the NLD for ${ }^{56}$ Fe nucleus was extracted in the framework of the statistical Hauser-Feshbach formalism of nuclear reactions.

In this paper, we investigate the NLDs and RSFs for ${ }^{56} \mathrm{Fe}$ and ${ }^{57} \mathrm{Fe}$ isotopes using three different experiments. These experiments and their results are discussed separately in the following Chapter. Finally concluding remarks are given in Chapter 3.

* $\quad$ Electronic address: tavukcu@ogu.edu.tr 


\section{EXPERIMENTS}

\section{1. ${ }^{57} \mathrm{Fe}\left({ }^{3} \mathrm{He},{ }^{3} \mathrm{He} \gamma\right){ }^{57} \mathrm{Fe}$ and ${ }^{57} \mathrm{Fe}\left({ }^{3} \mathrm{He}, \alpha \gamma\right){ }^{56} \mathrm{Fe}$ reactions}

The experiment was performed at the Oslo Cyclotron Laboratory with a $45-\mathrm{MeV}{ }^{3} \mathrm{He}$ beam on a $94.7 \%$ isotopically enriched ${ }^{57} \mathrm{Fe}$ target. The charged particles and $\gamma$ rays were detected in $8 \mathrm{Si} \triangle \mathrm{E}$-E telescopes placed $45^{\circ}$ with respect to the beam direction and 28 collimated $5^{\prime \prime} \times 5^{\prime \prime} \mathrm{NaI}(\mathrm{Tl})$ detectors, respectively. Details of the experiment are given in Ref. [1]. Known $Q$ value and reaction kinematics allow one to convert the ejectile energy into the initial excitation energy of the residual nucleus. Using the particle- $\gamma$ coincidences, an excitation energy of the residual nucleus versus $\gamma$-ray energy matrix is constructed, then the total $\gamma$-ray spectra for each excitation energy bin are unfolded. Finally, a distribution of primary $\gamma$ rays depopulating each excitation energy bin is extracted using a subtraction method described in Ref. [2].

Primary $\gamma$ rays are then factorized into a $\gamma$-ray transmission coefficient $T\left(E_{\gamma}\right)$, which depends only on the $\gamma$-ray energy $E_{\gamma}$, and into the level density $\rho\left(E-E_{\gamma}\right)$ using Brink-Axel hypothesis [3]. However this hypothesis has been proven to be voilated at sufficiently high temperatures. Models based on Fermi-liquid theory suggest a $T^{2}$ temperature dependence of the width of the GDR as well as the $\gamma$-ray energy $[4,5]$. In the present experiment, a rather low excitation energy range is covered, therefore, we assume that temperature changes very weakly within this excitation energy window. Thus, the actual temperature dependence in $T\left(E_{\gamma}, T\right)$ can be replaced with a constant average temperature.

The $T\left(E_{\gamma}\right)$ and $\rho\left(E-E_{\gamma}\right)$ are determined using a least $\chi^{2}$ fit to the experimental primary $\gamma$ matrix. Due to the functional form of the factorization, however, there is not a unique solution. One can find the relation between all of the solutions as follows:

$$
\begin{aligned}
\tilde{\rho}\left(E-E_{\gamma}\right) & =A \exp \left(\alpha\left(E-E_{\gamma}\right)\right) \rho\left(E-E_{\gamma}\right) \\
\tilde{T}\left(E_{\gamma}\right) & =B \exp \left(\alpha E_{\gamma}\right) T\left(E_{\gamma}\right)
\end{aligned}
$$

The free parameters $A$ and $\alpha$ are determined by normalizing the solution to the discrete levels and the neutron resonance spacing data. The parameter $B$ is determined using the average total radiative width of neutron resonances [1]. The normalized level densities for ${ }^{56,57} \mathrm{Fe}$ are shown in Fig. 1. The pronounced step structures in the level density curves 
are shown by arrows in Fig. 1. The discrete level data follow the experimental data quite well. Fluctuations in the discrete level curves are due to the binning procedure. These step structures in the level densities are explained as breaking of nucleon Cooper pairs. One can tentatively explain these steps using a simple microscopic model. The model considers 8 particles scattered into 8 doubly degenerate equidistant levels with Hamiltonian:

$$
\hat{H}=\epsilon \sum_{i=1}^{8} i a_{i}^{\dagger} a_{i}-\frac{1}{2} G \sum_{i, j=1}^{8} a_{i}^{\dagger} a_{i}^{\dagger} a_{j} a_{j}-\frac{1}{2} \kappa \sum_{i, j, k, l=1}^{8} W_{i j k l} a_{i}^{\dagger} a_{j}^{\dagger} a_{k} a_{l},
$$

where $a$ and $a^{\dagger}$ are Fermion creation and annihilation operators, respectively. The single particle level spacing $\epsilon$, the strength $G$ of the pairing interaction and the strength $\kappa$ of the seniority nonconserving interaction $W$ are the only parameters of the model. If there is pure pairing, i.e. $\kappa=0$, the distribution of levels with excitation energy gives individual bumps containing many levels with the same seniority as shown in Fig. 2.

For the sake of simplicity, we model the seniority nonconserving term with a random twobody interaction. The details of the calculations can be found in Ref. [1]. Individual bumps with the same seniority are smeared out by adding the random interaction which smooths the gap between the bumps. The best qualitative agreement between the model and experimental data are obtained for $\epsilon=0.25 \mathrm{MeV}, G=0.5 \mathrm{MeV}$, and $\kappa=0.14 \mathrm{MeV}$ (pairing +random interaction).

The radiative strength functions for ${ }^{56,57} \mathrm{Fe}$ obtained from the Oslo method are shown in Fig. 3. The most striking feature of the RSFs is an unusual enhancement for soft $\gamma$ rays. This feature can be described neither by a Lorentzian extrapolation of the GEDR nor the Kadmenskii-Markushev-Furman model [4] which includes a temperature dependence of the GEDR width as shown in Fig. 3. One can describe the RSFs phenomenologically as a sum of a renormalized KMF model, Lorentzian descriptions of the GMDR and the isoscaler E2 resonance, and a power law modeling the large enhancement at low energies:

$$
f_{\sum}=K\left(f_{E 1}+f_{M 1}+\frac{A}{3 \pi^{2} c^{2} \hbar^{2}} E_{\gamma}^{-B}\right)+E_{\gamma}^{2} f_{E 2}
$$

The parameters of the RSF models are taken from systematics [9]. The fit parameters for ${ }^{57} \mathrm{Fe}$ are $K=2.1(2), A=0.47(7) \mathrm{mb} / \mathrm{MeV}$, and $B=2.3(2)\left(E_{\gamma}\right.$ in $\left.\mathrm{MeV}\right)$.

Furthermore, the RSFs extracted from different excitation energy windows also prove that total RSFs do not depend on excitation energy. In order to ensure the low energy 
enhancement in the total RSFs, we performed two additional experiments which use different reactions. These experiments are described in the next two Chapters.

\section{2. ${ }^{57} \mathrm{Fe}(n, 2 \gamma){ }^{57} \mathrm{Fe}$ reaction}

The two step cascade (TSC) experiment was performed at the dual-use cold-neutron beam facility at the Budapest Research Reactor. After thermal neutron capture, the nucleus populates ground or one of the low lying levels by subsequent two $\gamma$ rays. The TSC spectrum of the unresolved doublet of the $1 / 2^{-}$ground state and the $3 / 2^{-}$first excited state at 14 $\mathrm{keV}$ are used for the analysis. Spectra to other final levels were not investigated due to their lower statistics and higher background. See details in Ref. [10]. The sequence of the two $\gamma$ rays is not determined experimentally in the TSC spectrum. In order to separate the soft primary and soft secondary $\gamma$ rays, we use the fact that spacing of the soft, discrete secondary transitions in low-energy region is considerably larger compared to the detector resolution. Thus, soft secondary transitions appear as discrete peaks on a continuum while the soft primary $\gamma$ rays create a continuous contribuion. Therefore, one can obtain the soft primary transitions by separating these discrete peaks from the TSC spectra in the appropriate energy interval. The experimental TSC intensities are shown with data points in Fig. 4.

TSC intensities can also be calculated theoretically using the statistical model of $\gamma$ decay from compound states:

$$
I_{i f}\left(E_{1}, E_{2}\right)=\sum_{X L, X L^{\prime}, J_{m}^{\pi}} \frac{\Gamma_{i m}^{X L}\left(E_{1}\right)}{\Gamma_{i}} \rho\left(E_{m}, J_{m}^{\pi}\right) \frac{\Gamma_{m f}^{X L^{\prime}}\left(E_{2}\right)}{\Gamma_{m}}
$$

where $E_{1}$ and $E_{2}$ are the energies of the first and second transition in the TSC which are connected by $E_{i}-E_{f}=E_{1}+E_{2} . \Gamma_{i m}$ and $\Gamma_{m f}$ are partial decay widths and $\Gamma_{i}$ and $\Gamma_{m}$ are total decay widths of the initial and intermediate $(m)$ levels, respectively. The average values of these widths can be calculated from the RSFs by

$$
\Gamma_{i f}^{X L}\left(E_{\gamma}\right)=\frac{f_{X L}\left(E_{\gamma}\right) E_{\gamma}^{2 L+1}}{\rho\left(E_{i}, J_{i}^{\pi}\right)}
$$

Summing in Eq. 4 is performed over all valid combinations of multipolarities $X L$ and $X L^{\prime}$ of transitions and of spins and parities of intermediate states. In the calculation of the TSC 
spectra, the statistical model employs the level density and RSFs which are extracted from the Oslo experiment.

Four calculations were performed: i) by neglecting the soft pole, ii) by assuming E1, iii) $E 2$, and iv) $M 1$ multipolarity for the soft pole in Eq. 3. These calculated TSC intensities are shown in Fig. 4. The calculations without the soft pole does not produce the experimental TSC intensities. However, one cannot infer the multipolarity of the soft pole due to the Porter-Thomas fluctuations of TSC intensities.

\section{3. ${ }^{55} \mathrm{Mn}(d, n){ }^{56}$ Fe reaction}

The last experiment was performed at John Edwards Accelerator Laboratory using 7-MeV deuteron beam on ${ }^{55} \mathrm{Mn}$ target. The energy of the outgoing neutrons is determined by the time-of-flight method. The experimental details of the experiment are given in Ref. [11]. The neutron spectra were measured at nine different angles from $20^{\circ}$ to $150^{\circ}$ using the beam swinger facility. The angular distribution of outgoing neutrons is shown in Fig. 5. The observed anisotropy at the forward angles less than $\approx 70^{\circ}$ represents contributions due to preequilibrum and direct reactions. The flat shaped angular distribution at backward angles is assumed to be due to the compound nuclear mechanism. Therefore the neutron spectra from the backward angles are used to extract the nuclear level density of the ${ }^{56} \mathrm{Fe}$ nucleus.

The neutron evaporation spectra can be described by a simple model based on HauserFeshbach theory of compound nuclear reactions [12]. According to this model the shape of the particle evaporation spectrum depends on the nuclear level density of the residual nucleus and the transmission coefficients of the outgoing particles, and the particle emission cross section is described as:

$$
\frac{d \sigma}{d \varepsilon_{b}}\left(\varepsilon_{a}, \varepsilon_{b}\right)=\sum_{J \pi} \sigma^{C N}\left(\varepsilon_{a}\right) \frac{\sum_{I \pi} \Gamma_{b}(U, J, \pi, E, I, \pi) \rho_{b}(E, I, \pi)}{\Gamma(U, J, \pi)}
$$

with

$\Gamma(U, J, \pi)=\sum_{b^{\prime}}\left(\sum_{k} \Gamma_{b^{\prime}}\left(U, J, \pi, E_{k}, I_{k}, \pi_{k}\right)+\sum_{I^{\prime} \pi^{\prime}} \int_{E_{c}}^{U-B_{b^{\prime}}} d E^{\prime} \Gamma_{b^{\prime}}\left(U, J, \pi, E^{\prime}, I^{\prime}, \pi^{\prime}\right) \rho_{b^{\prime}}\left(E^{\prime}, I^{\prime}, \pi^{\prime}\right)\right)$

where $\sigma^{C N}$ is the fusion cross section, $\rho_{b}(E, I, \pi)$ is the nuclear level density of the residual nucleus, $\varepsilon_{a}$ and $\varepsilon_{b}$ are energies of relative motion for incoming and outgoing channels, $\Gamma_{b}$ 
is the transmission coefficient of the outgoing particle, and $(U, J, \pi)$ and $(E, I, \pi)$ are the energy, angular momentum, and parity of the compound and residual nuclei, respectively, $E_{c}$ is the continuum edge.

Neutron transmission coefficients are calculated from the optical model potentials taken from the RIPL-2 data base [13]. The level density of ${ }^{56} \mathrm{Fe}$ is determined by chosing a nuclear level density model and then by adjusting the parameters of the model to reproduce the differential cross section of Eq. 6. The extracted level density is also normalized using the known discrete levels, and is shown in Fig. 6. The level density agrees very well with the counting of discrete levels up to $\approx 6 \mathrm{MeV}$ excitation energy as shown in the top panel in Fig. 6. Then the density of discrete levels drops due to missing levels, while the level density obtained from our experiment continues to increase. In the lower panel in Fig. 6, the NLD extracted from the neutron evaporation spectra and the NLD obtained from the Oslo experiment are compared. There is a very good agreement between the two NLDs up to $\approx$ $8 \mathrm{MeV}$, they start to diverge above this energy reaching about \%50 at an excitation energy of $\approx 8.8 \mathrm{MeV}$. This deviation may be due to the violations of the underlying assumptions of the two methods.

In general, considering that these two methods use different underlying assumptions, different reactions, and different analysis techniques, the extracted NLDs agree quite well. This implies that the statistical mechanism dominates in both reactions.

Due to the questions with the applicability of the Axel-Brink hypothesis employed in the Oslo method, it is valuable to extract the RSF by different means. With the NLD obtained from the neutron evaporation spectra and the primary $\gamma$ spectra obtained from the Oslo experiment, the RSF for ${ }^{56} \mathrm{Fe}$ can be extracted using the equation

$$
f\left(E_{\gamma}, E_{i}\right)=\frac{1}{2 \pi} \frac{N\left(E_{i}\right) P\left(E_{i}, E_{\gamma}\right)}{\rho\left(E_{i}-E_{\gamma}\right) E_{\gamma}^{3}}
$$

where $E_{i}=E_{\gamma}+E_{f}$. The extracted RSF shown in Fig. 7 agrees quite well with the RSF obtained from the Oslo experiment. This is due to the good agreement between the NLDs extracted by the two methods. From the comparison of these RSFs, one can conclude that the temperature effects on the RSF are very small compared to total uncertainties in the experimental data. Thus the Axel-Brink hypothesis employed in the Oslo method is applicable within the accuracy of the experimental data. 


\section{SUMMARY AND CONCLUSIONS}

Three different experiments have been performed in order to investigate ${ }^{56} \mathrm{Fe}$ and ${ }^{57} \mathrm{Fe}$ isotopes. The first experiment uses ${ }^{57} \mathrm{Fe}\left({ }^{3} \mathrm{He}, \alpha \gamma\right)$ reactions and ${ }^{57} \mathrm{Fe}\left({ }^{3} \mathrm{He},{ }^{3} \mathrm{He}^{\prime} \gamma\right)$ and helps to measure the NLDs and RSFs for the ${ }^{56} \mathrm{Fe}$ and ${ }^{57} \mathrm{Fe}$ isotopes with the use of Axel-Brink hypothesis. The unusual enhancement for $\gamma$ energies below $\approx 4 \mathrm{MeV}$ in the RSFs has not been observed before and furthermore cannot be explained by the current theoretical models. This raises questions about the applicablity of the Axel-Brink hypothesis used in the analysis. Therefore, a two step cascade experiment, ${ }^{57} \mathrm{Fe}(n, 2 \gamma){ }^{57} \mathrm{Fe}$ reaction, was done in order to further study the RSF for the ${ }^{57} \mathrm{Fe}$ isotope to address this concern. The observed TSC intensities also proves the enhacement in the low energy region of the RSF. In a third experiment, the NLD for ${ }^{56} \mathrm{Fe}$ is extracted from the neutron evaporation spectrum of the ${ }^{55} \mathrm{Mn}(d, n)^{56} \mathrm{Fe}$ reaction. This NLD is compared to that obtained from the Oslo experiment, and a fairly good agreement has been found, indicating the consistency of these two methods. Furthermore, the agreement between the RSF for the ${ }^{56} \mathrm{Fe}$ nucleus from the latter and the first experiments indicates the validity of the Axel-Brink hypothesis used in the Oslo method within the experimental errors.

\section{ACKNOWLEDGEMENTS}

Part of this work was performed under the auspices of the U.S. Department of Energy by the University of California, Lawrence Livermore National Laboratory under Contract W7405-ENG-48. Financial support from the Norwegian Research Council (NFR) is gratefully acknowledged. Part of this work was supported by the EU5 Framework Programme under Contract No. HPRI-CT-1999-00099. G.M., E.A., and U.A. acknowledge support by the U.S. Department of Energy Grant No. DE-FG02-97-ER41042. Part of this work was sponsored by the National Nuclear Security Administration under the Stewardship Science Academic Alliances program through DOE Research Grants No. DE-FG03-03-NA00074 and No. DEFG03-03-NA00076. 
1. A. Schiller et al., Phys. Rev. C 68, 054326 (2003).

2. M. Guttormsen et al., Nucl. Instrum. Methods Phys. Res. A 255, 518 (1987).

3. D.M. Brink, Ph.D. thesis, Oxford University, 1955; P. Axel, Phys. Rev. 126, 671 (1962).

4. S.G. Kadmenskii, V.P. Markushev, and V.I. Furman, Yad. Fiz. 37, 277 (1983) [Sov. J. Nucl. Phys. 37, 165 (1983)].

5. V.K. Sirotkin, Yad. Fiz. 43, 570 (1986) [Sov. J. Nucl. Phys. 43, 362 (1986)].

6. R. Fisher et al., Phys. Rev. C 30, 72 (1984).

7. T. von Egidy, H.H. Schmidt, and A.N. Bekhami, Nucl. Phys. A481, 189 (1988).

8. R.B. Firestone and V.S. Shirley, Table of Isotopes, 8th ed. (Wiley, New York, 1996), Vol. II.

9. P. Oblozinski, IAEA Report No. IAEA-TECDOC-1034, 1998.

10. A. Voinov et al., Phys. Rev. Lett. 93, 142504 (2004).

11. A. Voinov et al., Phys. Rev. C, , in press (2006).

12. H. Vonach, Proceedings of the IAEA Advisory Group Meeting on Basic and Applied Problems of Nuclear Level Densities, Upton, NY, 1983, BNL Report No. BNL-NCS-51694, 1983, p. 247.

13. T. Belgya et al., Handbook for calculations of nuclear reaction data: Reference Input Parameter Library, Vienna, 2005. 


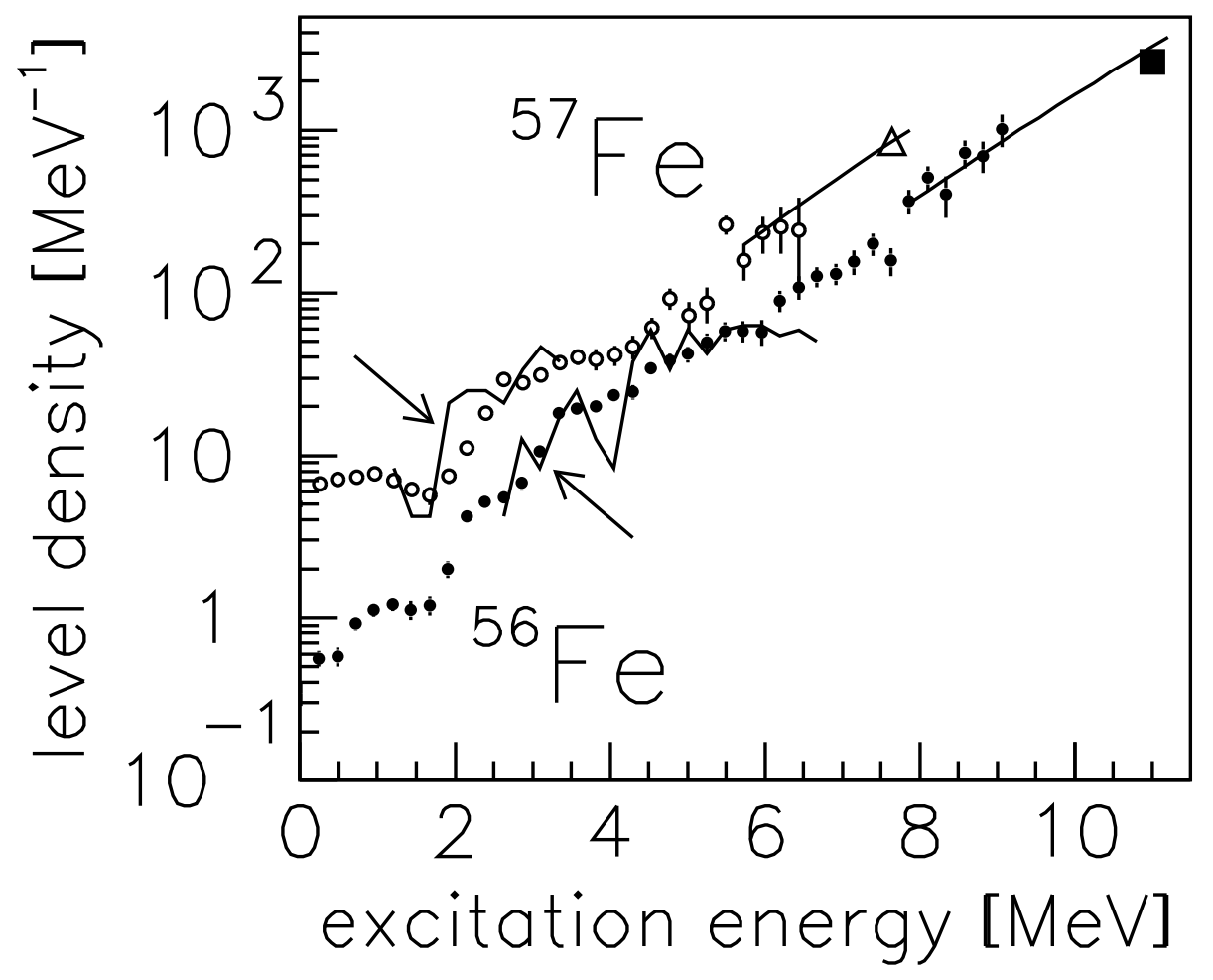

Figure 1. Experimental level densities of ${ }^{56,57} \mathrm{Fe}$. Full and open cicles represent ${ }^{56} \mathrm{Fe}$ and ${ }^{57} \mathrm{Fe}$, respectively. The square represents level density data from particle evaporation study [6]. Smooth solid curves are the renormalized level density parametrizations according to von Egidy et al.[7]. The jagged solid lines are level density information from counting of discrete levels [8]. Step structures in the level densities are marked by arrows. 


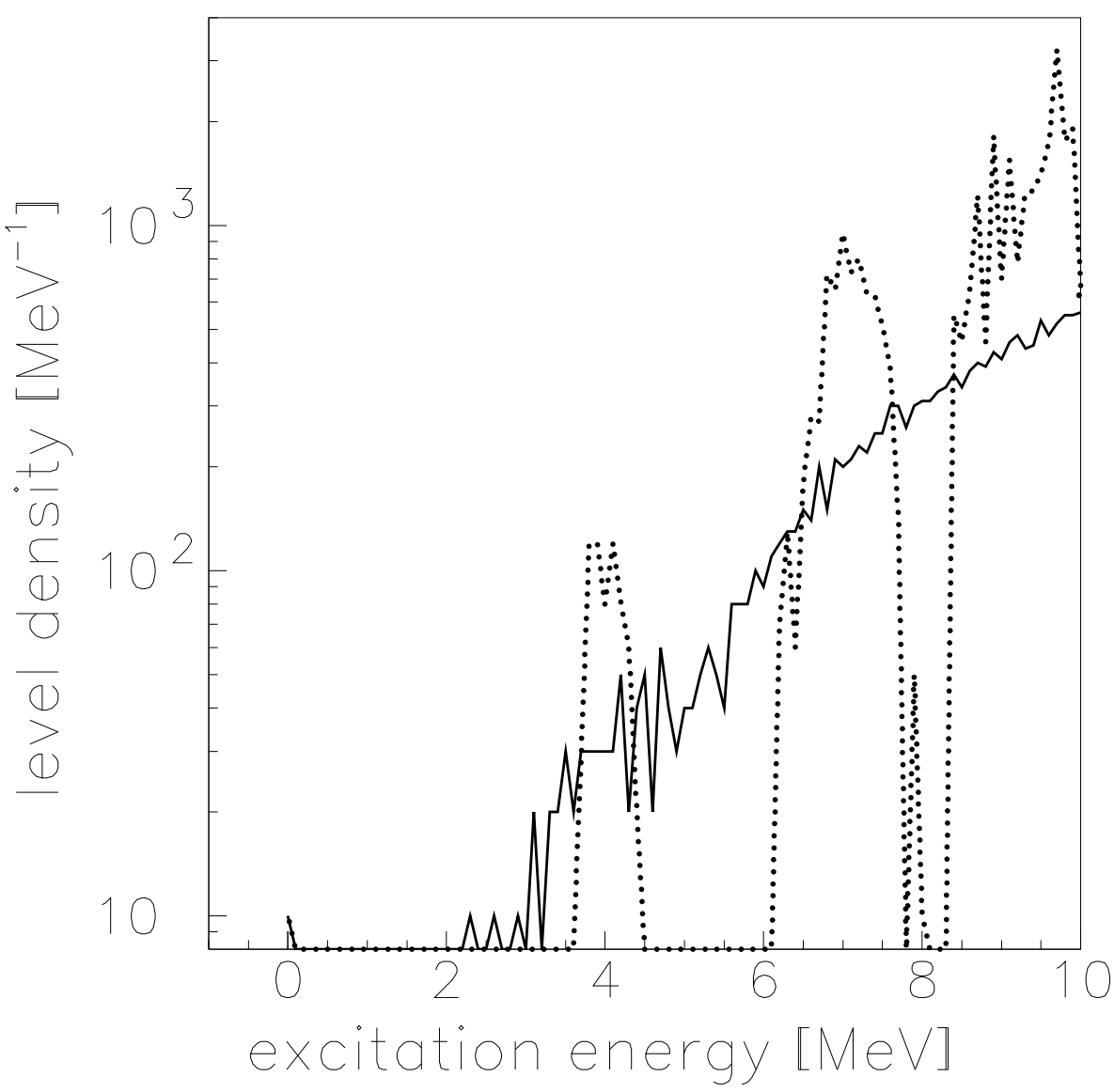

Figure 2. Level density obtained from the model calculations. Individual bumps represent many levels with the same seniority. Adding a random two-body interaction in the model Hamiltonian results in a step structure similar to the experimental level density curve in the iron isotopes. 

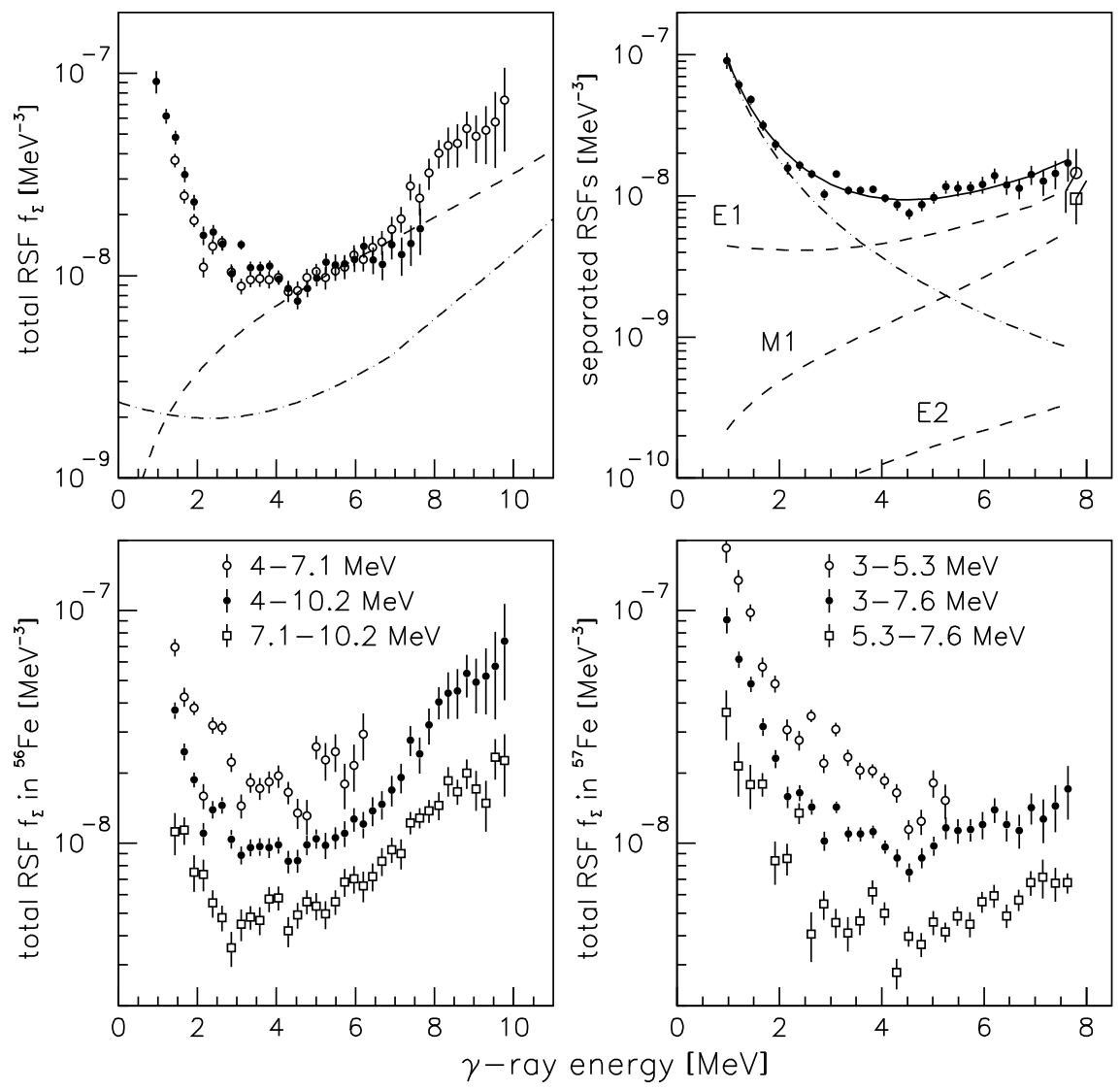

Figure 3. Upper left panel: Total RSF of ${ }^{56,57} \mathrm{Fe}$ (solid and open circles, respectively); Lorentzian (dashed line) and KMF model (dash-dotted line) descriptions of the GEDR. Upper left panel: Fit (solid line) to ${ }^{57} \mathrm{Fe}$ data and decomposition into the renormalized E1 KMF model, Lorentzian $M 1$ and $E 2$ models (all dashed lines), and a power law to model the large enhancement for low energies (dashed-dotted line). Lower panels: Total RSF in ${ }^{56} \mathrm{Fe}$ (left) and ${ }^{57} \mathrm{Fe}$ (right) for different excitation energy windows. 


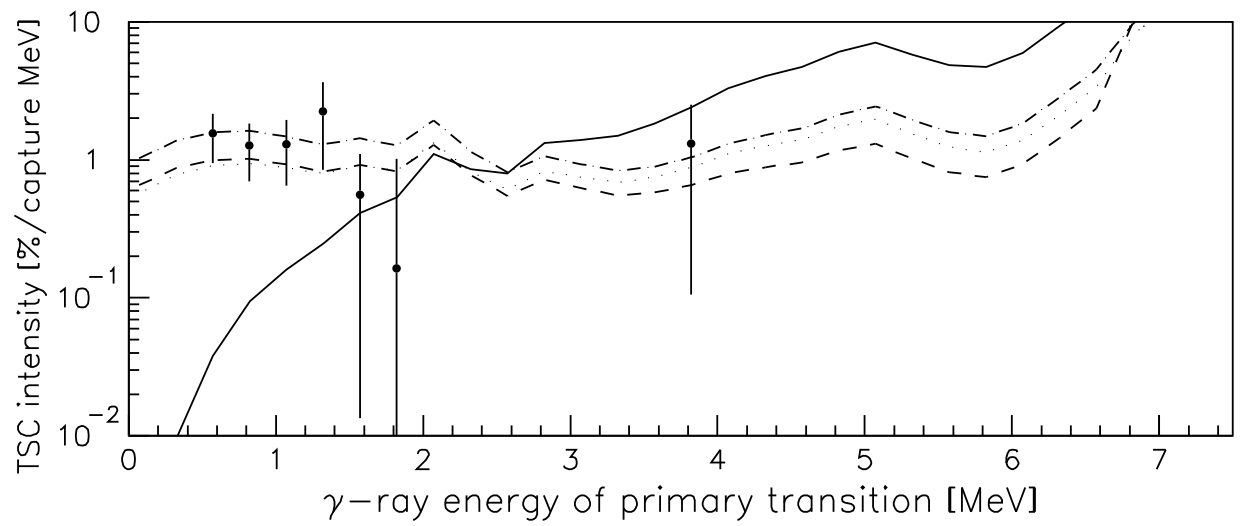

Figure 4. Experimental TSC intensities (compressed to 250-keV-broad $\gamma$ energy bins) for cascades with soft primary $\gamma$ rays and at the midpoint of the spectrum (data points with error bars). Lines are statistical model calculations based on experimental data for the level density and RSF, neglecting (solid line) and assuming E1 (dashed line), $M 1$ (dash-dotted line), and E2 (dotted line) multipolarity for the soft pole of the RSF. 


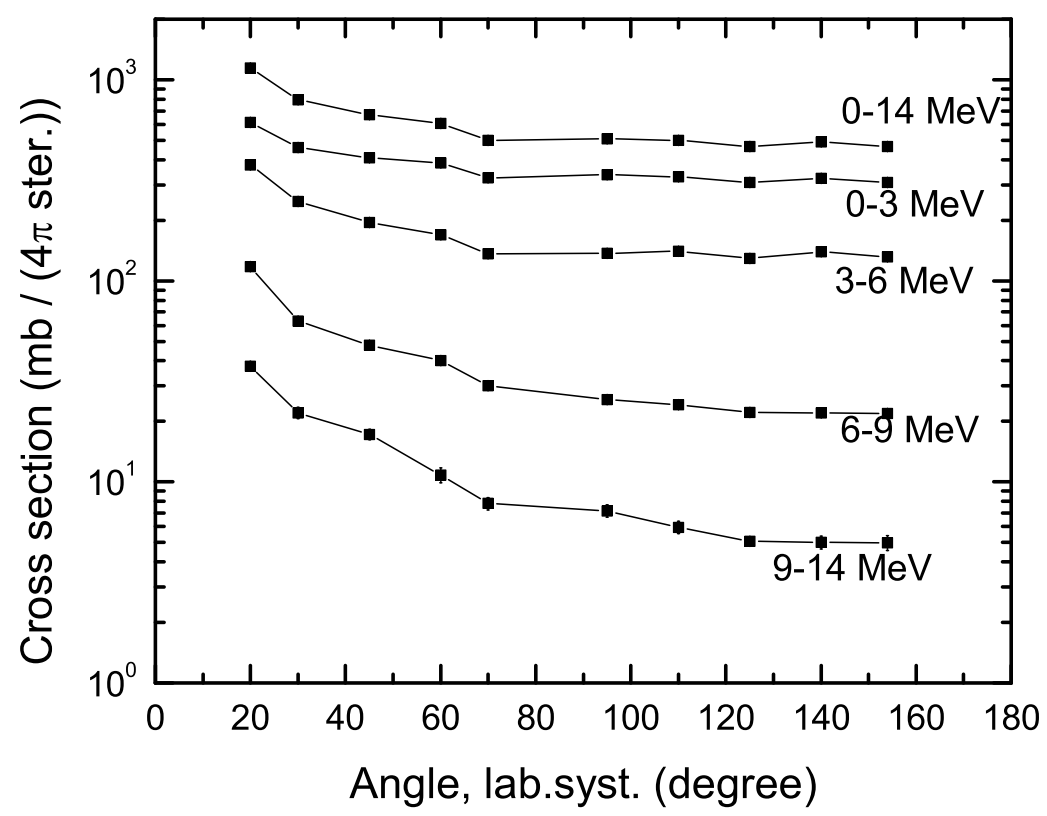

Figure 5. Angular distribution for different energy groups of outgoing neutrons from ${ }^{55} \mathrm{Mn}(d, n)$ reaction. 


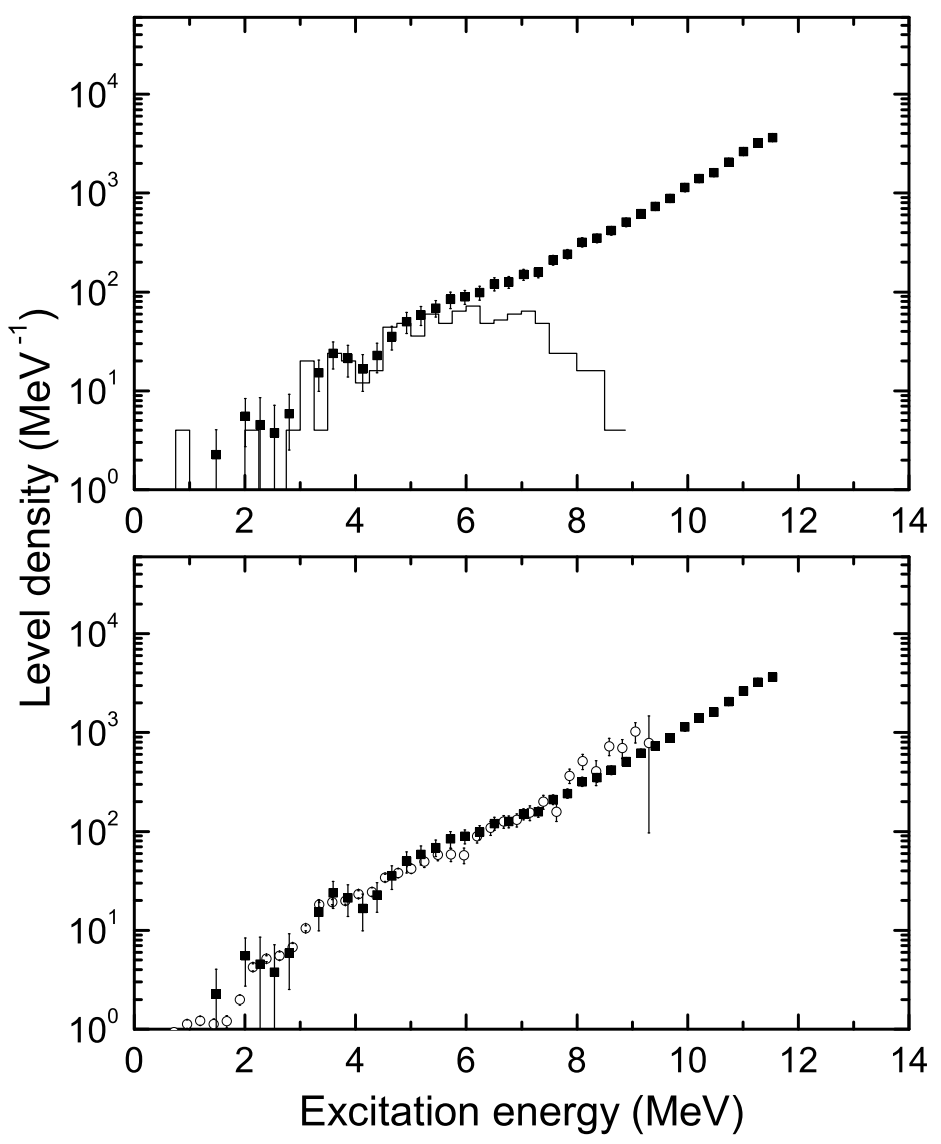

Figure 6. Comparison of the nuclear level density extracted from the neutron evaporation spectra (full circles) with discrete levels (upper panel) and with the level density obtained from the Oslo experiment (lower panel). 


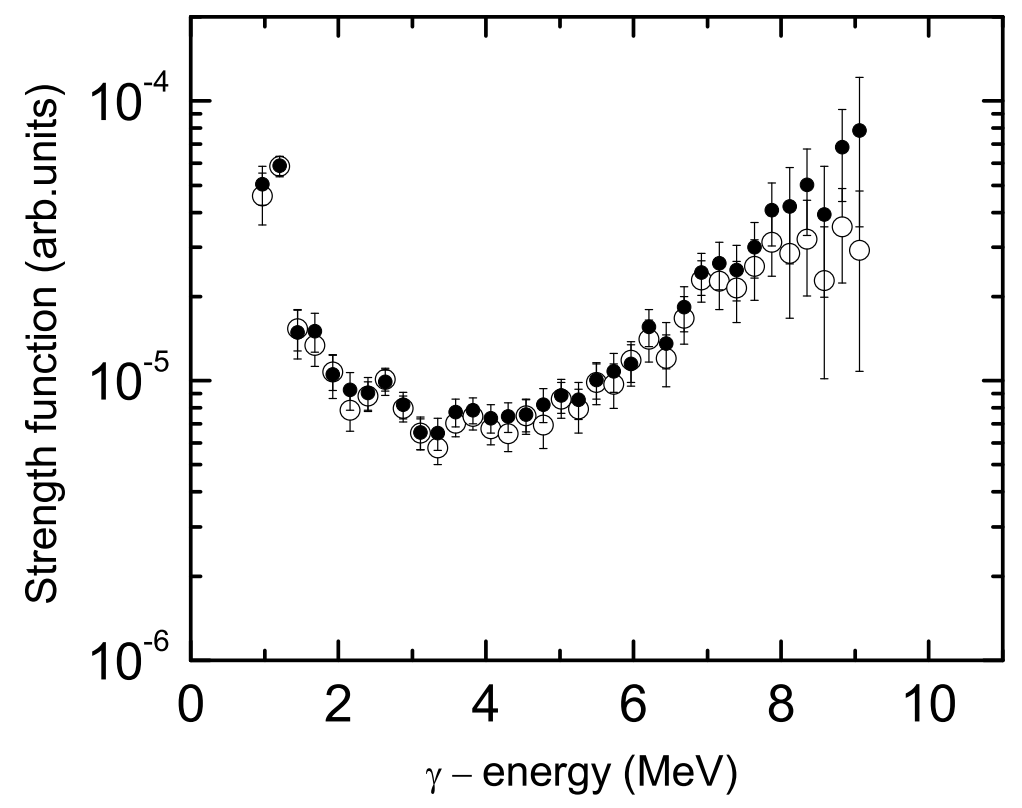

Figure 7. The RSF extracted using Eq. 8 with the NLD from the neutron evaporation spectra and the primary $\gamma$ spectra obtained from the Oslo experiment (filled circles), and the RSF obtained from the Oslo experiment (open circles). 